\title{
A Novel Low Cost Planar RFID Miniature Antenna
}

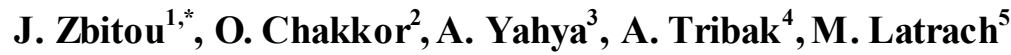 \\ ${ }^{1}$ LITEN, Faculté Polydiscip linaire de KHOURIBGA FPK, université Hassan $1^{\mathrm{er}}$, Morocco \\ ${ }^{2}$ ENSA Tetouan. Univ. Abdelmalek Essaadi Tetouan, Morocco \\ ${ }^{3}$ Faculty of Sciences. Univ. Abdelmalek Essaadi Tetouan, Morocco \\ ${ }^{4} \mathrm{INPT}$, Groupe d'Electronique et Instrumentation à la faculté des sciences de Tétouan, Morocco \\ ${ }^{5}$ Ecole supérieure d'Electronique de l'Ouest, BP 30926, 49009 Angers, France
}

\begin{abstract}
This paper presents a new miniature low cost planar RFID antenna designed @ 2.45 GHz. In order to validate the finalstructure, we have conducted a study on two antenna structures. The first one is based on a rectangular patch with a centred slot line, and the second one is based on a monopole antenna. By comparing the performances ofboth structures we have chosen the second one because due to the fact that presents a miniature dimensions and especially due to their compactness and robustness against the mechanical tolerances. The antenna achieved and measured demonstrates a good agreement between simulation and measurement results. Moreover, for improving the matching input impedance of the antenna, we have studied and optimized all geo metry dimensions of the whole structure. For the feed line we have used a CPW feed which permits to integrate this antenna easily with the memory circuit to form a tag system.
\end{abstract}

Keywords RFID, Microstrip Antennas, ISM Band

\section{Introduction}

The market for radio frequency identification (RFID) technology is growing rapidly. RFID is a method that allows to remotely retrieve, store, and man ipulate data contained

in a transponder unit that is permanently attached to an object. At present, RFID systems are used in identifying various products, containers and others applications [1],[2],[3],[4],[5]. Examp les of applications include: animal tagging, asset tracking, electronic passports, smartcards and shop security.

The tag antenna is an essential circuit in passive or active transponders and RFID systems [6],[7],[8],[9],[10],[11],[12]. Therefore it is necessary to match the input impedance of the antenna to a load in order to efficiently couple the RF power. Among The big challenges today considering the applications of the RFID tags which demand a miniature tag system, is to decrease dimensions of the tag by doing a good choice of the architecture of the antenna as sociated to the chip memory. Among the frequency band for RFID applications we find the ISM" Industrial Scientific Band" at $2.45 \mathrm{GHz}[13],[14],[15],[16],[17],[18]$.

In this work, we have conducted a study of two planar topologies of antennas which can be combined with a microchip to form a tag circuit. Therefore we have optimized the both circu its and compared there performances

* Corresponding author:

zbitou.jamal@yahoo.fr (J. Zbitou)

Published online at http://journal.sapub.org/eee

Copyright (C) 2012 Scientific \& Academic Publishing. All Rights Reserved and dimensions. By the end, we have chosen the miniature structure which is a CPW feed low cost monopole planar antenna.

\section{Design and Simulation Procedure}

In this part we will introduce and describe the comparis on study of two planar RFID antennas at $2.45 \mathrm{GHz}$. The aim is to choice by the end a miniature, compact and low cost structure that can be integrated easily to a microchip memory.

\subsection{The First structure}

To define the real dimensions of a rectangular patch at 2.45 GHz, we have simulated a rectangular patch, with FR4 as substrate with a thickness of $1.58 \mathrm{~mm}$ and 4.4 for the dielectric permittivity. As shown in Figure. 1, the dimensions of the antenna are $37.54 \mathrm{~mm} \mathrm{X} 28.12 \mathrm{~mm}$.

The Figure. 2 presents the return loss of the antenna with a good matching input impedance at $2.45 \mathrm{GHz}$.

In bibliography, many techniques were developed to miniature the tag antennas[19],[20],[21],[22],[23][24],[25]. In this work, we have started this work by developing a new technique to miniature a rectangular patch antenna. As shown in Figure.3, by using ADS “Advanced Design System” we have optimized the dimensions of the patch by introducing a slot line in the antenna structure.

Table. 1 presents the different dimensions of the new structure. As a conclusion of this first study we can deduce that we have validated a new technique permitting to decrease the RFID antenna dimensions at $2.45 \mathrm{GHz}$. 
The aim of this work is to validate a structure that can be miniature and easily integrated with microchip circuits, which push us to develop a new CPW feed antenna structure in the ISM frequency band.

Table 1. Dimensions in $\mathrm{mm}$

\begin{tabular}{|c|c|c|c|c|c|c|c|c|}
\hline $\mathrm{a}$ & $\mathrm{b}$ & $\mathrm{c}$ & $\mathrm{d}$ & $\mathrm{e}$ & $\mathrm{f}$ & $\mathrm{g}$ & $\mathrm{h}$ & $\mathrm{i}$ \\
\hline 0.97 & 1 & 9.75 & 1.5 & 11.75 & 0.416 & 11.66 & 1.53 & 8.33 \\
\hline
\end{tabular}

\subsection{The Second Structure}

The final designed antenna structure is based on a CPW feed and a monopole antenna which is presented in Figure.4. After many simulations of the antenna circuit by using ADS optimization method we have validated the final circu it with the dimensions presented in table.2:

Table 2. Dimensions in $\mathrm{mm}$

\begin{tabular}{|c|c|c|c|c|c|c|c|c|}
\hline $\mathrm{a}$ & $\mathrm{b}$ & $\mathrm{c}$ & $\mathrm{d}$ & $\mathrm{e}$ & $\mathrm{f}$ & $\mathrm{g}$ & $\mathrm{h}$ & $\mathrm{La}$ \\
\hline 1 & 5.3 & 8.28 & 2.02 & 1.94 & 10 & 0.71 & 9.59 & 8 \\
\hline
\end{tabular}

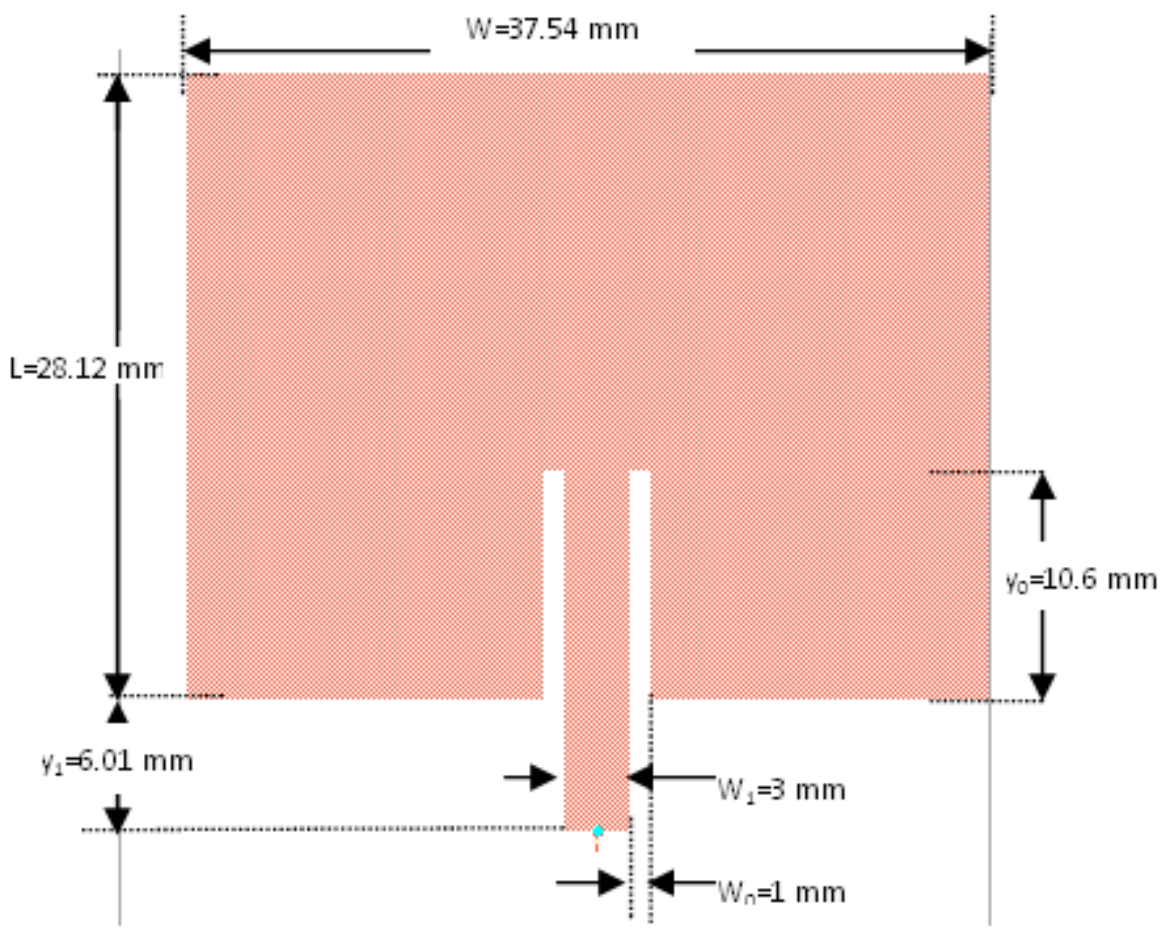

Figure 1. Rectangular patch structure

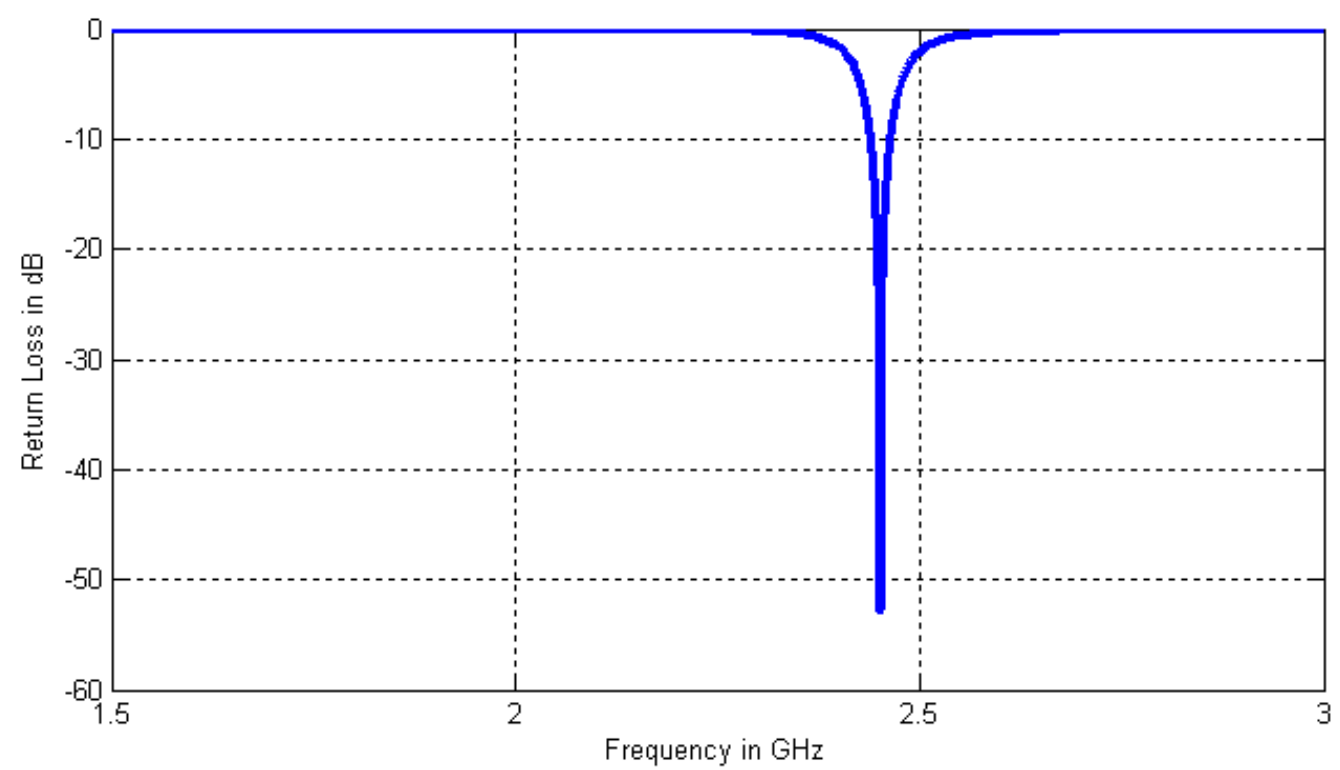

Figure 2. The return loss of the rectangular patch antenna 


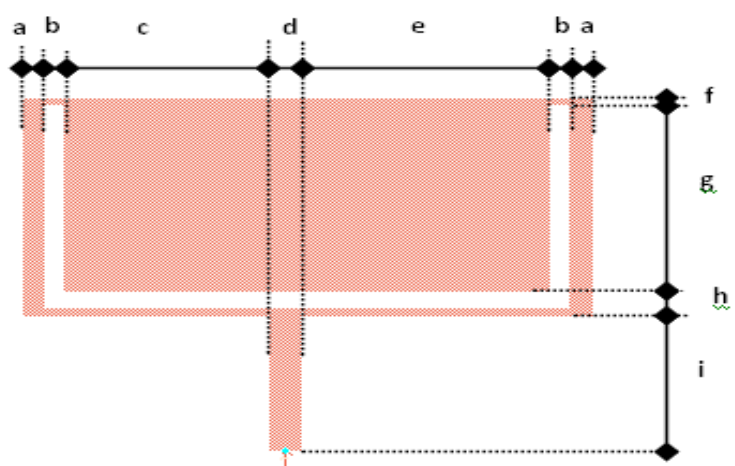

Figure 3. Rectangular patch structure with a slot line

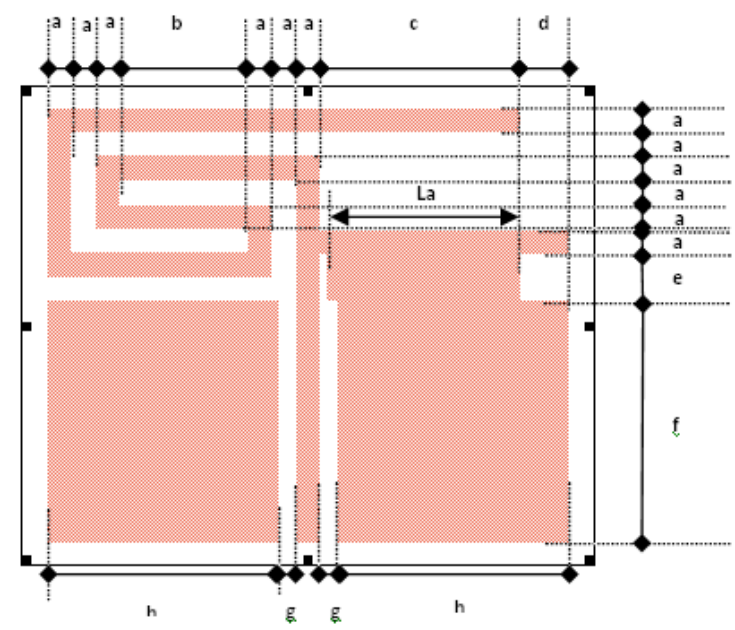

Figure 4. CPW feed Monopole antenna

As shown in Figure.5 (a), the antenna operates in the ISM band at $2.45 \mathrm{GHz}$ with $-24 \mathrm{~dB}$ as a return loss presenting good matching input impedance. For the radiation pattern it is presented in Figure.5 (b). To improve the matching input impedance we have lunched a study on the different parameters that can influence the return loss at the operating frequency. After many series of simulations we have deduced that the La parameter is the key to enhance the return loss as demonstrated in Figure.6. By consequent, To improve the matching input impedance we have to increase the length of La:

$S 11$

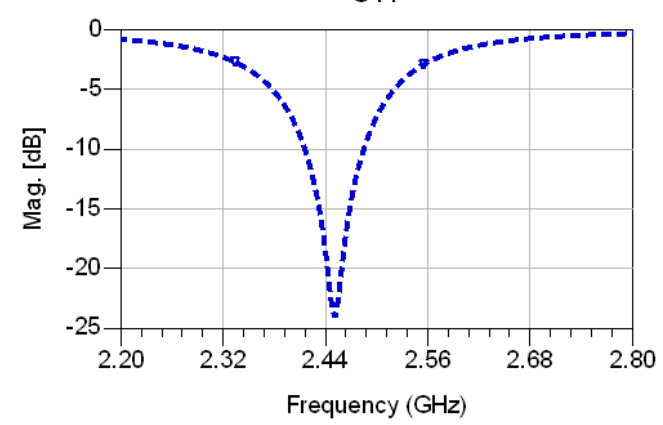

(a)

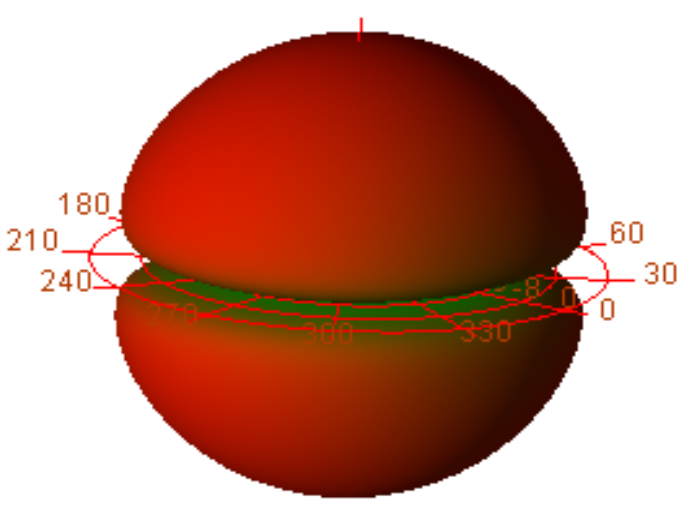

(b)

Figure 5. a- The return loss vs. frequency b-The radiation pattern @ 2.45 $\mathrm{GHz}$

The antenna designed presents $-70 \mathrm{MHz}$ as a bandwidth with a center frequency of $2.45 \mathrm{GHz}$. As comparison between the second antenna structure and the first one we can conclude that the second circuit presents a miniature and compact structure. Due to the CPW feed of this antenna it will facilitate its integration to the microchip memory which permits to achieve at the end a miniature tag.

\section{Results and Discussion}

As shown in Figure.7 (a) the antenna was measured by using VNA "vector network analy zer" R\&S®ZVB20. When using a VNA to perform antenna measurements, a careful calibration has to be conducted.

The reasons are:

- As a radiator, the antenna should not be placed too close to the VNA to avoid coupling and interference, that is, it should not be directly connected to the VNA. Thus, a cable or connectors have to be used.

- The cable and connectors introduce attenuation and a phase shift.

- The reading on the VNA is at the default reference plane, but what we want to measure is the reading at the input port of the antenna.

We therefore need to remove the effects of the cable or connectors and shift the measurement reference plane right to the end of the cable. The standard calibration needs three terminations for one-port calibration, i.e. short, open and

Load-matched. The calibration which we have used is the $3.5 \mathrm{~mm}$ Agilent technologies calibration Kit.

Figure. 7 (b) shows the simu lated and measured return loss results of the antenna which are in agreement. The measured return loss is less than $-20 \mathrm{~dB}$ at the operating frequency 2.45 $\mathrm{GHz}$. 


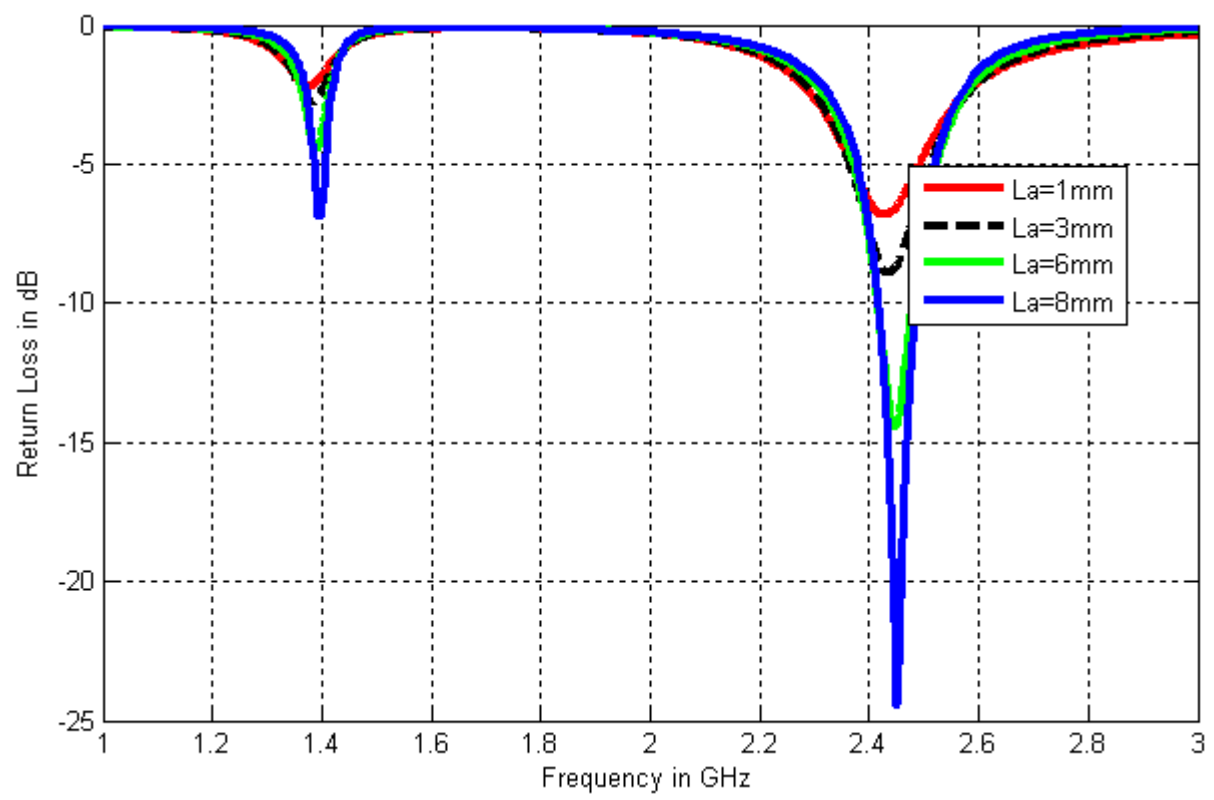

Figure 6. The influence of $\mathrm{La}$ on the return loss

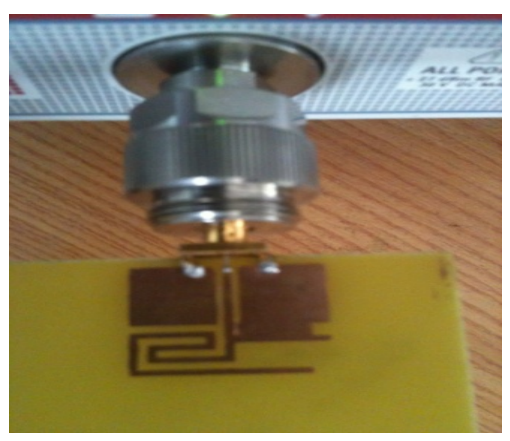

(a)

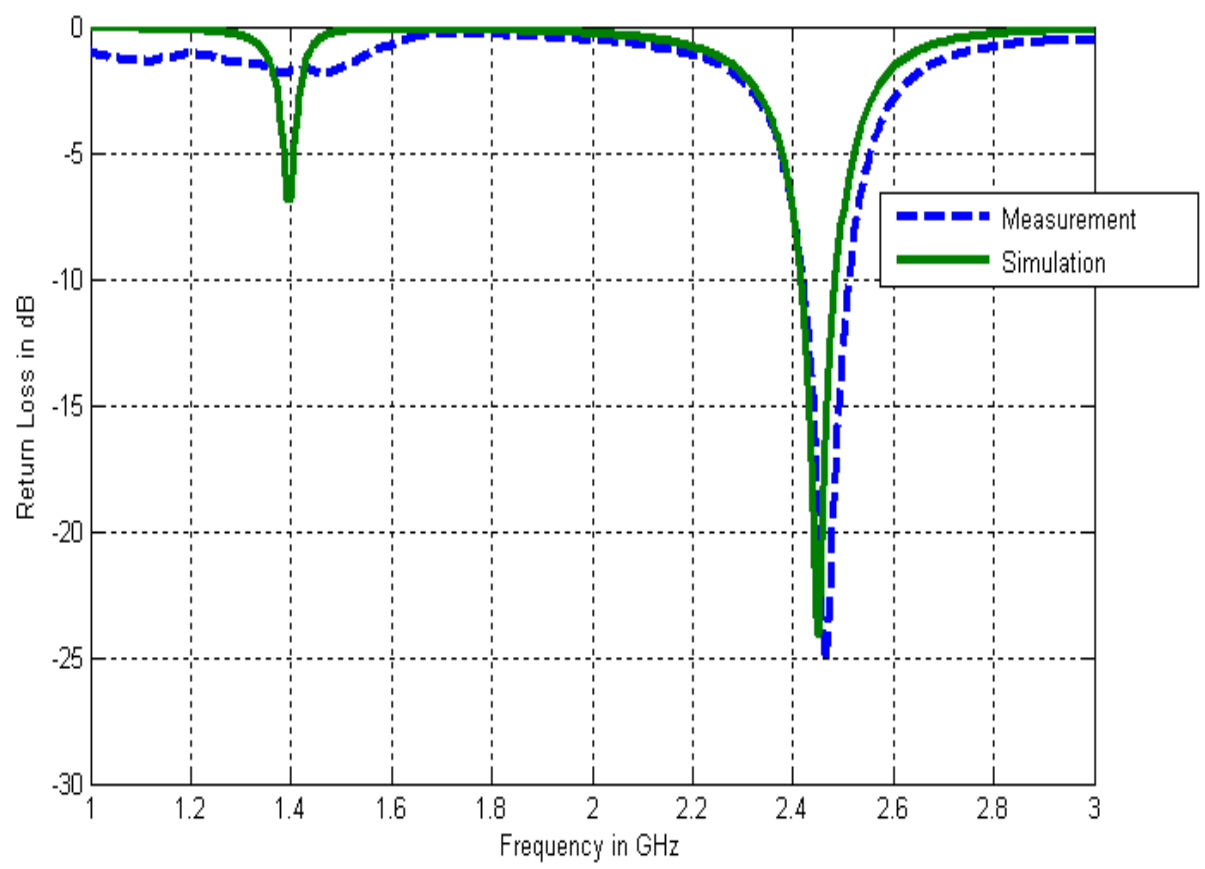

(b)

Figure 7. a. The antenna prototype achieved b. Measured and simulated return loss 


\section{Conclusions}

A miniature CPW feed RFID monopole antenna has been developed in the ISM band at an operating frequency equal to $2.45 \mathrm{GHz}$. Before this validation, we have proposed two studies which describe the methodology and procedure followed to miniature the antenna dimensions. The first antenna validated into simulation can also be used for ISM band applications but taking into account the goal of our work that is the achievement of planar antenna which can be integrated easily with a microchip to form a tag circuit, we have optimized and defined a novel antenna structure. The antenna designed is validated into simulation and measurements after many optimizations that permit to validate a novel RFID miniature low cost planar antenna.

\section{REFERENCES}

[1] K.Ahsan, H.Shah and P. Kingston "RFID Applications: An Introductory and Exploratory Study" IJCSI International Journal of Computer Science Issues, Vol. 7, Issue 1, No. 3, January 2010, pp 1-7.

[2] E.ILIE-Zudor, Z.Kemeny, P.Egri “The RFID Technology and its current Applications" proceedings of The Modern Information Technology in the Innovation Processes of the Industrial Enterprises-MITIP 2006, pp29-36.

[3] Seshagirl Rao, K. V., P. V. Nikitin, and S. F. Lam, “Antenna design for UHF RFID tags: A review and a practical application," IEEE Trans. Antennas Propagat., Vol. 53, No. 12, 3870-3876, Dec.2005.

[4] L. Ukkonen, L. Sydanheimo, and M. Kivikoski, "A Novel Tag Design Using Inverted-F Antenna for Radio Frequency Identification of Metallic Objects," Proc. 2004 IEEE/Sarnoff Symp. Advances in Wired and Wireless Communication, Apr. 2004, pp. 91-94.

[5] K. V. Seshagiri Rao, Pavel V. Nikitin, and Sander F. Lam "Antenna Design for UHF RFID Tags: A Review and a Practical Application,“ IEEE Transactions on antennas and propagation, vol. 53, NO. 12, December 2005.

[6] C. Chang, and Y. C. Lo, "Broadband RFID tag antenna with capacitively coupled structure" Electron Lett., vol. 42, pp. 1322-1323,Nov. 2006.

[7] R. Want, "An introduction to RFID technology" IEEE Pervasive Comput., vol. 5, no. 1, pp. 25-33, Jan.-Mar. 2006.

[8] Texas Instruments, Application Report March 2005 "ISM-Band and Short Range Device Antennas".

[9] Nasimuddin, Chen, Z.N. and Qing, X. (December2010), Asymmetric-circular shaped slotted microstrip antennas for circular polarization and RFID applications, IEEE Trans. Antennas and Propagation, Vol.58, No.12, pp.3821-3828, 2010

[10] L. Mats, J. T. Cain, M. H. Mickle, "The In-Situ Technique for Measuring Input Impedance and Connection Effects of RFID Tag Antenna", IEEE Transactions on Automation Science and Engineering, vol. 6, no. 1, 2009, pp. $4-8$
[11] J. Griffin, G. Durgin, A. Haldi, B. Kippelen, "RF Tag Antenna Performance on Various Materials Using Radio Link Budgets", IEEE AWP Letters, vol. 5, no. 1, Dec. 2006, pp. $247-250$

[12] P. V. Nikitin and K. V. S. Rao, "LabVIEW-based UHF RFID tag test and measurement system", IEEE Transactions on Industrial Electronics, vol. 56, no. 7, pp. 2374-2381, July 2009

[13] A. Kamerman and N. Erkocevic, "Microwave oven interference on wireless LANs operating in the 2.4-GHz ISM band," in Proc. 8th IEEE Int. Symp. Personal, Indoor and Mobile Radio Communications, Helsinki, Finland, Sept. 1997, pp. 1221-1227.

[14] Carla-Fabiana Chiasserini, Member, IEEE, and Ramesh R. Rao" Coexistence Mechanis ms for Interference Mitigation in the 2.4-GHz ISM Band" IEEE Transactions on Wireless Communications, vol. 2, NO. 5, SEPTEMBER 2003

[15] W. J. Krzysztofik, "M odified Sierpinski Fractal Monop ole for ISM-Bands Handset Applications", IEEE Transaction on Antennas and Propagation, vol. 57, no. 3,pp. 606-615, Mar. 2009.

[16] I-Fong Chen, Chia-Mei Peng and Sheng-Chieh Liang. 2005. "Single Layer Printed Monopole Antenna for Dual ISM-Band Operation". IEEE Transactions on antennas and propagation. Vol.53(4): 1270-1273.

[17] IEEE Std. 802-11, "IEEE Standard for Wireless LAN Medium Access Control (MAC) and Physical Layer (PHY) Specification," June 1997.

[18] Institute of Electrical and Electronics Engineers. IEEE Std 802.11-2007,Wireless LAN Medium Access Control (MAC) and Physical Layer (PHY) Specifications, 12 June 2007.

[19] R. Bhattacharyya, C. Floerkemeier, S. Sarma, "Low-Cost, Ubiquitous RFID-Tag-Antenna-Based Sensing", Proceed ings of the IEEE, vol. 98 , no. 9, 2010 , pp. $1593-1600$

[20] Marrocco, G., IThe art of UHF RFID antenna design: Impedance-matching and size-reduction techniques," IEEE Antennas and Propag. Magazine, Vol. 50, No. 1, 66\{79, Feb. 2008.

[21] A. Ibrahiem, T. P. Vuong, A. Ghiotto, and S. Tedjini, "New design antenna for RFID UHF tags," in Proc. IEEE AP-S'06, 2006, p. 1355-1358.

[22] L. Ukkonen, M. Schaffrath, D. W. Engels, L. Sydanheimo, and M. Kivikoski, "Operability of folded microstrip patch-type tag antenna in the UHF RFID bands within 865-928 MHz," IEEE Antennas Wireless Propag. Lett., vol. 5, pp. 414-417, Dec. 2006.

[23] Ahmed Toaha Mobashsher, Mohammad Tariqul Islam and Norbahiah Misran. 2010. "A Novel High-Gain Dual-Band Antenna for RFID Reader Applications". IEEE antenna and wireless propagation letters. 9: pp 653-656.

[24] Yong Cai, Y. Jay Guo and Andrew R. Weily. 2010 “A Novel High-Gain Dual-Band Antenna for RFID Reader Applications". IEEE antenna and wireless propagation letters. 9: 883-886.

[25] H. W. Son, J. Yeo, G. Y. Choi, and C. S. Pyo, \A low-cost, wideband antenna for passive RFID tags mountable on metallic surfaces," in Proc. IEEE Antennas and Propagation Society 